\title{
APLICAÇÃO DE BIOFILME EM FRUTOS DE CIRIGUELA: EFEITO NA CONSERVAÇÃO E NO POTENCIAL ANTIOXIDANTE
}

\author{
DOUGLAS SEIJUM KOHATSU* \\ BEATRIZ CERVEJEIRA BOLANHO** \\ VALDIR ZUCARELI*** \\ KELLY CAROLINE GENARO**** \\ FABIANA BREMM MARTINS ${ }^{* * * * *}$
}

\begin{abstract}
A ciriguela é um fruto climatério que possui vida de prateleira limitada, a qual pode ser aumentada com a utilização de biofilme. Esta tecnologia associada à refrigeração pode auxiliar na manutenção dos compostos antioxidantes presentes. O objetivo deste trabalho foi avaliar a utilização de cobertura de fécula de mandioca nas características físico-químicas e na capacidade antioxidante de frutos de ciriguela verdes durante o armazenamento refrigerado. Os frutos selecionados e sanitizados foram separados em 2 grupos: controle e recoberto com biofilme de fécula de mandioca a $3 \%$. Durante 9 dias de armazenamento foram avaliados os parâmetros: coloração, teor de sólidos solúveis (SS), compostos fenólicos totais e atividade antioxidante pelo método ABTS. Notou-se que a aplicação de cera possibilitou melhor preservação dos compostos fenólicos e da atividade antioxidante dos frutos, além de retardar o aumento do teor de SS durante o armazenamento. O chilling causado pelo resfriamento dos frutos foi amenizado pela utilização de biofilme. Portanto, o tratamento utilizado prolongou a vida útil dos frutos de ciriguela submetidos à refrigeração e auxiliou na manutenção do teor de compostos antioxidantes.
\end{abstract}

*Doutor em Agronomia, Professor, Departamento de Ciências Agrárias, Universidade Estadual de Maringá, Umuarama, PR (e-mail: dskohatsu2@uem.br)

**Doutora em Ciência de Alimentos, Professora, Departamento de Tecnologia, Universidade Estadual de Maringá, Umuarama, PR (e-mail: beatrizbolanho@yahoo.com.br)

${ }^{* * *}$ Doutor em Ciências Biológicas, Professor, Departamento de Ciências Agrárias, Universidade Estadual de Maringá, Umuarama, PR (e-mail: valdirzucareli@yahoo.com.br)

****Graduada em Tecnologia em Alimentos, Departamento de Tecnologia, Universidade Estadual de Maringá, Umuarama, PR (e-mail: kelly_genaro@yahoo.com.br)

*****Graduada em Agronomia, Departamento de Ciências Agrárias, Universidade Estadual de Maringá, Umuarama, PR (e-mail: bremmfbm@gmail.com) 


\section{INTRODUÇÃO}

Dentre as espécies do Gênero Spondias, a ciriguela (Spondias purpurea) é uma das que se destacam, sendo explorada de forma extrativista em várias regiões tropicais e subtropicais do mundo (Aroucha et al., 2012). Por apresentar frutos climatéricos, possui vida pós-colheita limitada, de modo que a adoção de técnicas para aumento da conservação da qualidade dos frutos é essencial para a expansão do período de oferta e comercialização após a colheita (VIEIRA et al., 2010). A utilização de embalagens que prolonguem a vida de prateleira de frutas e hortaliças é uma prática comum. Segundo Machado et al. (2012), o uso crescente dos polímeros sintéticos é uma questão ambiental de grande preocupação devido aos problemas de contaminação devido à sua não biodegradabilidade.

Segundo Vila et al. (2007), a utilização de fécula de mandioca pode representar uma alternativa potencial à elaboração de biofilmes a serem usados na conservação de frutas, a qual apresenta baixo custo e grande disponibilidade, o que facilita seu emprego. Além desta alternativa, Kohatsu et al. (2011) observaram que a refrigeração empregada de maneira adequada favorece o armazenamento de frutos de ciriguela, porém, se utilizada temperatura excessivamente baixa provoca aumento na taxa respiratória.

Além de aumentar a vida de prateleira há uma constante busca por técnicas que preservem os compostos bioativos presentes nos frutos. Perspectivas promissoras para exploração de frutos tropicais não tradicionais se devem aos níveis consideráveis de vitamina $C$, antocianinas, carotenóides e compostos fenólicos, além da capacidade antioxidante destes frutos. A atividade antioxidante está relacionada a inúmeros benefícios à saúde humana por prevenir o efeito deletério dos radicais livres, espécies altamente reativas que são geradas no organismo humano (RUFINO et al., 2010).

Portanto, o desenvolvimento de técnicas de conservação em frutos de ciriguela se torna necessário, principalmente por meio da utilização de filmes comestíveis diferenciados como o de fécula de mandioca, que são de extrema importância por auxiliarem na preservação dos frutos e por não prejudicarem o meio ambiente. $O$ aumento da vida útil da ciriguela permite sua comercialização em locais distantes do centro produtor e possibilita agregar valor a cadeia produtiva.

O objetivo deste trabalho foi avaliar a eficiência da atmosfera modificada na vida de prateleira e na manutenção da capacidade antioxidante de frutos de ciriguela verdes armazenados sob refrigeração.

\section{MATERIAL E MÉTODOS}

Os frutos de ciriguela foram obtidos em vários pontos da região de Umuarama/PR e transportados em caixas isotérmicas para o Laboratório de Processamento de Produtos Vegetais da Universidade Estadual de Maringá, Campus de Umuarama-PR, onde foram selecionados quanto à aparência, estádio de maturação e ausência de danos físicos e sanitizados em solução de 50 $\mathrm{mg} \mathrm{L}^{-1}$ de cloro livre. Em seguida, os frutos verdes foram recobertos com biofilme de fécula de mandioca (BFM) $3 \%$, o qual foi preparado por aquecimento com agitação da suspensão até $70^{\circ} \mathrm{C}$ para a geleificação da fécula. Após a imersão dos frutos por 1 minuto nesta solução, procedeu-se a secagem natural, sendo posteriormente acondicionados em bandejas de poliestireno expandido e armazenados em temperatura de $8^{\circ} \mathrm{C} \pm 1^{\circ} \mathrm{C}$ e umidade de $90 \% \pm 5 \%$.

O delineamento experimental utilizado foi inteiramente casualizado em esquema fatorial 2 x 4 (com e sem aplicação de biofilme x tempo de armazenamento) totalizando 8 tratamentos com 3 repetições, com 100 gramas de frutos por parcela. Para todas as variáveis os dados obtidos foram submetidos à análise de variância e comparação das médias utilizando teste Tukey ao nível de $5 \%$ de probabilidade. As avaliações foram realizadas a cada três dias durante 9 dias (0, 3, 6, e 9 dias) de acordo com os seguintes parâmetros: teor de compostos fenólicos, capacidade antioxidante, coloração (luminosidade e ângulo Hue) e sólidos solúveis.

A extração dos compostos antioxidantes dos frutos de ciriguela verdes foi realizada de acordo com Hung et al. (2009), utilizando etanol $80 \%$ e a quantificação de compostos fenólicos 
totais pelo método Folin-Ciocalteau, conforme descrito por Bucic-Kojic et al. (2007). A concentração total de fenóis de cada extrato foi quantificada por meio de uma curva padrão preparada com ácido gálico e expresso como mg de equivalente de ácido gálico $100 \mathrm{~g}^{-1}$ de amostra (mg EAG $100 \mathrm{~g}^{-1}$ ).

A capacidade antioxidante dos extratos frente ao radical livre ABTS $^{+}$foi realizada de acordo com Thaipong et al. (2006). Os resultados foram expressos como capacidade antioxidante equivalente ao Trolox ( $\mu \mathrm{mol}$ de Trolox $\mathrm{g}$ de amostra $^{-1}$ ).

A determinação da cor foi executada com auxílio de colorímetro Konica Minolta (Chroma Meter-400). Na colorimetria de reflexão, o valor $L^{*}$ significa luminosidade em um eixo de 0 a 100 (do preto ao branco), enquanto os valores do ângulo Hue $\left({ }^{\circ} \mathrm{h}\right.$ ) representam o verde (entre $100^{\circ} \mathrm{e} 180^{\circ}$ ), amarelo (entre $70^{\circ}$ e $100^{\circ}$ ), vermelho (entre $25^{\circ}$ e $70^{\circ}$ ) e azul (entre $0^{\circ}$ e $25^{\circ}$ ) (Ding et al., 2007). As leituras foram realizadas em lados opostos de um mesmo fruto.

Os teores de sólidos solúveis (SS) foram determinados na polpa por refratometria em refratômetro tipo ABBE (Atago-N1) a $25^{\circ} \mathrm{C}$, e os resultados foram expressos em ${ }^{\circ} \mathrm{Brix}$.

\section{RESULTADOS E DISCUSSÃO}

O teor de compostos fenólicos e o potencial antioxidante frente ao radical ABTS.+ pode ser observado na Tabela 1. Notou-se que os teores de fenólicos totais na ciriguela armazenada durante os 9 dias foram maiores que o valor encontrado por Almeida et al. (2011) em ciriguela madura (55 $\left.\mathrm{mg} 100 \mathrm{~g}^{-1}\right)$. Segundo os mesmos autores o teor destes compostos em frutos exóticos brasileiros

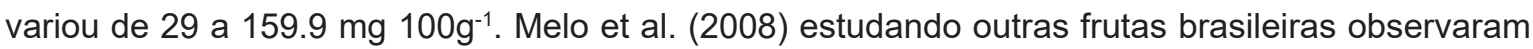
valores entre 25,7 e $496,2 \mathrm{mg} 100 \mathrm{~mL}^{-1}$ de fenólicos totais. Wang \& Lin (2000) observaram que o teor de fenólicos totais em morango, framboesa e amora diminuíram conforme o aumento do tempo de armazenamento do fruto, como também observado neste estudo.

Durante o armazenamento, houve redução no teor de compostos fenólicos em $53 \%$ nos frutos de ciriguela verdes e sem aplicação de cera, e de $17 \%$ nos recobertos com a cera, consequentemente este grupo manteve maior potencial antioxidante (PA) no período de estocagem avaliado. $\mathrm{O}$ uso de atmosfera modificada (cera) contribuiu para manter os compostos antioxidantes, possivelmente pela diminuição da concentração de oxigênio, composto responsável por provocar reações de oxidação que geram radicais livres. Houve uma correlação significativa $(r=0,96 ; p<0,05)$ entre o teor de fenólicos totais e o PA dos frutos verdes armazenados sob refrigeração durante 9 dias, demonstrando que estes compostos são grandes responsáveis pela interação com radicais livres, tornando-os inativos.

Os valores de atividade antioxidante dos frutos verdes de ciriguela foram próximos ao encontrado por Severo et al. (2010) em frutos de physalis imaturos (1,7 $\mu \mathrm{mol}$ de Trolox g-1 de amostra). Almeida et al. (2011) relataram valores entre 0,63 a 15,73 $\mu \mathrm{M}$ de Trolox $\mathrm{g}^{-1} \mathrm{em}$ frutos exóticos brasileiros.

O resfriamento dos frutos verdes provocou o "chilling", principalmente em frutos sem aplicação de cera (Figura 1), resultado da ação de enzimas oxidativas dependentes de oxigênio sobre os compostos fenólicos, provocando o escurecimento dos frutos, e consequentemente a diminuição no teor de fenólicos e no potencial antioxidante como observado neste experimento.

Em relação à luminosidade $\left(L^{*}\right)$, não houve diferença significativa entre os tratamentos e os dias de armazenamento, de modo que independente do tratamento, ocorreu manutenção dos valores para este parâmetro (Tabela 1). Embora não observado escurecimento durante o armazenamento para este experimento por meio de avaliação colorimétrica, houve tendência de maiores valores de luminosidade no tratamento com cera no último dia, ou seja, menor escurecimento. Kohatsu et al. (2011) observaram escurecimento em frutos de ciriguela armazenados a $25^{\circ} \mathrm{C}$ e atribuíram à maior atividade da enzima polifenoloxidase em sua temperatura ideal, porém, as pontuações negras nos frutos causadas pelo "chilling" foram encontradas em temperaturas de $4^{\circ} \mathrm{C}$, abaixo da temperatura utilizada neste experimento. Foi encontrado menor quantidade de pontuações ou a ausência em alguns frutos tratados com cera, provavelmente, devido a redução da disponibilidade de oxigênio que, por sua vez diminui a atividade das enzimas fenólicas oxidativas, principalmente a polifenoloxidase. 
TABELA 1 - TEOR DE COMPOSTOS FENÓLICOS TOTAIS (MG DE ÁCIDO GÁLICO 100G1), POTENCIAL ANTIOXIDANTE, FRENTE AO RADICAL ABTS.+ (MMOL DE TROLOX G-1 DE AMOSTRA), LUMINOSIDADE (L*), ÂNGULO HUE ('HUE) E SÓLIDOS SOLÚVEIS DE FRUTOS DE CIRIGUE-LA VERDES RECOBERTOS OU NÃO COM BIOFILME DURANTE O ARMAZENAMENTO

\begin{tabular}{|c|c|c|c|c|c|c|}
\hline \multirow{2}{*}{ Análise } & \multirow{2}{*}{ Tratamentos } & \multicolumn{4}{|c|}{ DIAS } & \multirow[t]{2}{*}{ CV } \\
\hline & & 0 & 3 & 6 & 9 & \\
\hline \multirow{2}{*}{$\begin{array}{c}\text { Fenólicos } \\
\text { totais }\end{array}$} & Sem biofilme & $150,8 \mathrm{aA}$ & 111,9 bB & 100,6 bC & 70,9 bD & \multirow{2}{*}{$3,38^{* *}$} \\
\hline & Com biofilme & $150,8 \mathrm{aA}$ & $147,2 \mathrm{aA}$ & $123,1 \mathrm{aB}$ & $125,1 \mathrm{aB}$ & \\
\hline \multirow{2}{*}{$\mathrm{ABTS}^{+}$} & Sem biofilme & $1,85 \mathrm{aA}$ & $1,47 \mathrm{bB}$ & $1,11 \mathrm{bC}$ & $0,74 \mathrm{bD}$ & \multirow{2}{*}{$7,36^{* *}$} \\
\hline & Com biofilme & $1,85 \mathrm{aA}$ & $1,78 \mathrm{aA}$ & $1,65 \mathrm{aAB}$ & $1,41 \mathrm{aB}$ & \\
\hline \multirow{2}{*}{$\begin{array}{l}\text { Luminosidade } \\
\qquad\left(L^{*}\right)\end{array}$} & Sem biofilme & $50,59 \mathrm{aA}$ & 50,98 aA & $49,36 \mathrm{aA}$ & $52,59 \mathrm{aA}$ & \multirow{2}{*}{$4,07^{*}$} \\
\hline & Com biofilme & $49,38 \mathrm{aB}$ & $53,17 \mathrm{aAB}$ & $50,10 \mathrm{aB}$ & $56,07 \mathrm{aA}$ & \\
\hline \multirow{2}{*}{$\begin{array}{l}\text { Ângulo Hue } \\
\text { ('hue) }\end{array}$} & Sem biofilme & $172,8 \mathrm{aA}$ & $173,0 \mathrm{aA}$ & 87,6 bB & $76,3 \mathrm{bC}$ & \multirow{2}{*}{$1,29^{* \star}$} \\
\hline & Com biofilme & $172,8 \mathrm{aA}$ & $171,9 \mathrm{aA}$ & 170,9 aA & $167,24 \mathrm{aB}$ & \\
\hline \multirow{2}{*}{ ss } & Sem biofilme & $8,20 \mathrm{aC}$ & $10,70 \mathrm{aB}$ & $11,73 \mathrm{aB}$ & $14,80 \mathrm{aA}$ & \multirow{2}{*}{$5,73^{* \star}$} \\
\hline & Com biofilme & $8,20 \mathrm{aC}$ & $9,10 \mathrm{bBC}$ & $10,03 \mathrm{bB}$ & $13,10 \mathrm{bA}$ & \\
\hline
\end{tabular}

Letras minúsculas diferentes na mesma coluna e letras maiúsculas diferentes na mesma linha indicam diferença significativa* $(p<0,05)$ pelo Teste de Tukey. ${ }^{\text {ns }}$ ão significativo; * significativo a 0,05; ** significativo a 0,01

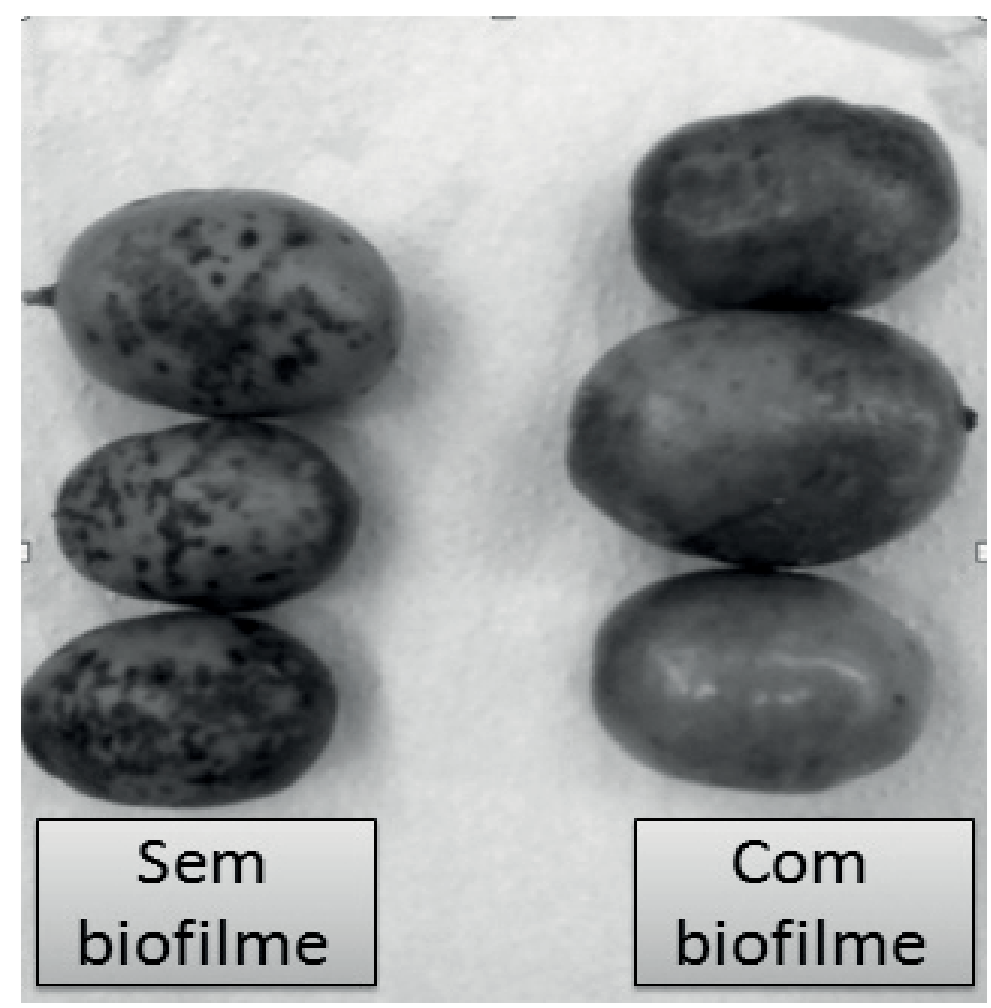

FIGURA 1 - FRUTOS DE CIRIGUELA COM (DIREITA) E SEM (ESQUERDA) APLICAÇÃO DE BIOFILME. 
De acordo com os dados obtidos para o teor de sólidos solúveis (SS) observa-se significância entre os tratamentos e os dias de armazenamento (Tabela 1). Ambos os tratamentos, com e sem aplicação de biofilme, apresentaram aumento para este parâmetro durante todo o período experimental, comportamento típico de frutos climatéricos. No entanto, neste tipo de fruto, após atingirem o máximo teor de SS ocorre redução dos mesmos, isso porque os açúcares constituem grande porcentagem dos sólidos solúveis que serão posteriormente consumidos no processo respiratório. Kohatsu et al. (2011) trabalharam com frutos de ciriguela em temperatura de $8^{\circ} \mathrm{C}$ e observaram o mesmo resultado durante os 8 dias de armazenamento, porém, quando armazenados a $25^{\circ} \mathrm{C}$ os frutos atingiram o máximo teor de sólidos solúveis no segundo dia de armazenamento, com redução desses teores até o último dia de análise.

Os frutos submetidos à aplicação de BFM apresentaram menores valores de SS a partir do terceiro dia de armazenamento até o último dia de análise, provavelmente, por reduzir a permeabilidade do oxigênio e, consequentemente, a taxa respiratória e o consumo de açúcares. Independentemente do tratamento, os resultados apresentados para o teor de SS estão dentro daqueles encontrados por Sampaio

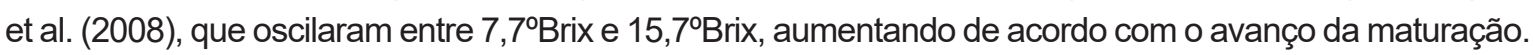

Por meio dos dados obtidos para o teor de SS, observa-se que nos frutos sem aplicação de cera houve amadurecimento acelerado em relação ao tratamento com cera. A maior velocidade de amadurecimento pode ser sustentada pelos resultados obtidos para o ângulo Hue (Tabela 1).

Nos frutos sem BFM houve mudança na coloração, de maneira que ao final do experimento os frutos apresentaram ângulos entre $70^{\circ}$ e $100^{\circ}$, na faixa de coloração amarelo, de modo que no último dia de análise estava próximo a faixa de coloração vermelha $\left(25^{\circ}\right.$ a $\left.70^{\circ}\right)$, caracterizando o avanço do amadurecimento. Contudo, o mesmo comportamento não foi observado para os frutos com cera, de modo que mantiveram valores na faixa de coloração verde, com ângulos sempre superiores a $160^{\circ}$, sustentando a hipótese de menor velocidade de amadurecimento dos frutos tratados com BFM. A diferença estatística para este parâmetro ocorre a partir do sexto dia de armazenamento, onde os valores de $\mathrm{o}$ foram superiores em frutos com BFM $3 \%$.

$\mathrm{O}$ atraso no amadurecimento pode ser atribuído a duas hipóteses baseadas na mudança de permeabilidade aos gases devido à utilização de cera. A primeira hipótese segue a linha de raciocínio aplicada ao menor consumo de açúcares devido à limitação do oxigênio no processo respiratório, composto que além de ser essencial para a respiração aeróbica, também participa ativamente na produção de etileno como co-fator da enzima ACC oxidase, sendo esse hormônio responsável pelo amadurecimento de frutos climatéricos. A segunda hipótese é a liberação de dióxido de carbono pela respiração dos frutos e, consequente seu acúmulo devido a menor permeabilidade do BMF, de modo que em altas concentrações inibem a atividade da ACC oxidase e ACC sintase, que de acordo com Winkler et al. (2002) necessitam de co-fatores como o dióxido de carbono e oxigênio para obterem atividade máxima.

\section{CONCLUSÃO}

O biofilme comestível de fécula de mandioca preservou as características físico-químicas dos frutos de ciriguela de maneira mais eficiente que o tratamento controle. Além disso, sua aplicação minimizou o efeito do 'chilling' causado durante $\mathrm{o}$ armazenamento à baixas temperaturas e auxiliou na manutenção do teor de compostos fenólicos totais e da capacidade antioxidante dos frutos.

\section{ABSTRACT}

\section{BIOFILM APPLICATION IN CIRIGUELA FRUITS: CONSERVATION AND ANTIOXIDANT POTENTIAL EFFECT}

The ciriguela is a climacteric fruit which has limited shelf life that can be increased by biofilm utilization. This technology associated with cooling may contribute to maintain the 
antioxidant compounds in the fruit. The aim of this work was to evaluate the cassava starch coat on physicochemical characteristics and antioxidant capacity in ciriguela fruits during cold storage. The fruits were selected, sanitized and separated into 2 groups: control and biofilm coated with cassava starch $3 \%$. During the storage (9 days) the parameters color, soluble solids (SS), total phenolics and antioxidant activity by ABTS method were evaluated. It was noted that biofilm application enabled a better preservation of phenolic compounds and antioxidant activity of the fruit and delayed the increase of SS during storage. The chilling caused in the fruits by cooling storage was minimized by biofilm utilization. Therefore, the treatment applied to ciriguela increased the shelf life of the fruits stored in refrigeration and preserved the antioxidant compounds.

\section{KEY-WORDS: SPONDIAS PURPUREA L.; BIOFILM; PHENOLIC COMPOUNDS; ANTIOXIDANTS; MATURATION}

\section{REFERÊNCIAS}

1 ALMEIDA, M. M. B.; SOUSA, P. H. M.; ARRIAGA, A. M. C.; PRADO, G. M.; MAGALHÃES, C. E. C.; MAIA, G. A.; LEMOS, T. L. G. Bioactive compounds and antioxidant activity of fresh exotic fruits from northeastern Brazil. Food Research International, v.44, p. 2155-2159, ago. 2011.

2 AROUCHA, E. M. M.; SOUZA, C. S. M.; SOUZA, A. E. D.; FERREIRA, R. M. A.; AROUCHA FILHO, J. C. Qualidade póscolheita de cajarana em diferentes estádios de maturação durante armazenamento refrigerado. Revista Brasileira de Fruticultura, v.34, n.2, p.391-399, jun. 2012.

3 BUCIC-KOJIC, A.; PLANINIC, M.; TOMAS, S.; BILIC, M.; VELIC, D. Study of solid-liquid extraction kinetics of total polyphenols from grape seeds. Journal of Food Engineering, v. 81, p. 236-242, jul. 2007.

4 DING, P.; AHMAD, S.H.; GHAZALI, H.M. Changes in select quality characteristics of minimally processe carambola (Averrhoa carambola L.) when treated with ascorbic acid. Journal of the Science of Food and Agriculture, v.87, p.702709, fev. 2007

5 HUNG, P. V.; MAEDA, T.; MIYATAKE, K.; MORITA, N. Total phenolic compounds and antioxidant capacity of wheat graded flours by polishing method. Food Research International, v. 42, p. 185-190, jan. 2009.

6 KOHATSU, D. S.; ZUCARELI, V.; BRAMBILLA, W. P.; EVANGELISTA, R. M.; ONO, E. O.; SILVA, T. R. B. Storage temperature and quality of red mombin fruits (Spondia purpureas L.). Journal of Food, Agriculture and Environment, v.9, n.3 e 4, p.132-134, jul. 2011.

7 MACHADO, B. A. S.; REIS, J. H. de O.; SOUZA, C. O.; SANTANA, M. C. C. B.; DRUZIAN, J. I. Tendências tecnológicas de embalagens biodegradáveis através da prospecção em documentos de patentes. Cadernos de Prospecção, v.5, n.3, p.132-140, nov. 2012.

8 MELO, E. A.; MACIEL, M. I. S.; GALVÃO DE LIMA, V. L. A.; NASCIMENTO, R. J. Capacidade antioxidante de frutas. Revista Brasileira de Ciências Farmacêuticas, v. 44, n. 2, 193-201, abr. 2008.

9 RUFINO, M. S. M.; ALVES, R. E.; BRITO, E. S.; PÉREZ-JIMÉNEZ, J.; SAURA-CALIXTO, F.; MANCINI-FILHO, J. Bioactive compounds and antioxidant capacities of 18 non-traditional tropical fruits from Brazil. Food Chemistry, v. 121, p. 9961002, ago. 2010.

10 SAMPAIO, S. A.; BORA, P. S.; HOLSCHUH, H. J. Postharvest respiration and maturation of some lesser-known exotic fruits from Brazil: Ciriguela (Spondias purpurea L.). Ceres, v.55, n.2, p.141-145, mar. 2008.

11 SEVERO, J.; LIMA, C. S. M.; COELHO, M. T.; RUFATTO, A. R.; ROMBALDI, C. V.; SILVA, J. A. Atividade antioxidante e fitoquímicos em frutos de physalis (Physalis peruviana, I.) durante o amadurecimento e o armazenamento. Revista Brasileira de Agrociência, v. 16, n.1-4, p. 77-82, jan. 2010.

12 THAIPONG, K.; BOONPRAKOB, U.; CROSBY, K; CISNEROSZEVALLOS, L.; BYRNE, D.H. Comparison of ABTS, DPPH, FRAP and ORAC assays for estimating antioxidant activity from guava fruit extracts. Journal of Food Composition and Analysis, v.19, p.669-675, set. 2006.

13 VILA, M. T. R.; LIMA, V. C. O.; VILAS BOAS, E. V. B.; HOJO, E. T. D.; RODRIGUES, L. J.; PAULA, N. R. F. Caracterização química e bioquímica de goiabas armazenadas sob refrigeração e atmosfera modificada. Ciência e Agrotecnologia, v.31, n.5, p.1435-1442, set. 2007.

14 WANG, S. Y.; LIN H-S. Antioxidant Activity in Fruits and Leaves of Blackberry, Raspberry, and Strawberry Varies with Cultivar and Developmental Stage. Journal of Agricultural and Food Chemistry, v.48, p.140-146, jan. 2000.

15 WINKLER, L. M.; QUOIRIN, M.; AYUB, R.; ROMBALDI, C.; SILVA, J. Produção de etileno e atividade da enzima ACCoxidase em frutos de maracujá-amarelo (Passiflora edulis f. flavicarpa Deg.). Revista Brasileira de Fruticultura, v.24, n.3, p.634-636, dez. 2002.

16 VIEIRA, M. J.; ARGENTA, L. C.; AMARANTE, C. V. T.; STEFFENS, C. A.; VIEIRA, A. M. F. D. Preservação da qualidade pós-colheita de kiwi 'bruno' pelo controle do etileno. Revista Brasileira de Fruticultura, v.32, n.2, p.397-406, jun. 2010. 